\title{
A NONOSCILLATION THEOREM FOR A NONLINEAR SECOND ORDER DIFFERENTIAL EQUATION
}

\author{
J. W. HEIDEL
}

In this paper we consider the real-valued solutions of the equation

$$
y^{\prime \prime}+q(t) y^{\gamma}=0
$$

where $q(t) \geqq 0$ and continuous on some half line $[a, \infty)$ and $\gamma$ satisfies $0<\gamma=p / q<1$ where $p, q$ are odd integers. Our purpose is to give conditions under which all solutions of (1) are nonoscillatory. The result we give is similar to that given by Atkinson [1] for the case $\gamma>1$ but the proof is different.

The restriction to $\gamma=p / q$ where $p$ and $q$ are odd is significant. For example, if $q$ is even and $p$ odd, then oscillatory solutions are not real-valued. If $p$ is even and $q$ odd, then all nonzero solutions are trivially nonoscillatory. Similar problems arise if $\gamma$ is irrational.

5 We begin with some definitions and basic facts. A solution of (1) is said to be extendable (continuable) if it exists on some half line $[b, \infty)$. Since $0<\gamma<1$, all solutions of (1) are extendable. This follows from a theorem of Wintner [3, p. 29]. A nontrivial solution of (1) is called oscillatory if it has arbitrarily large zeros. Otherwise, a solution is called nonoscillatory, i.e., if it is of one sign for large $t$. Since $\gamma$ is restricted to be odd, solutions with real initial conditions are real-valued and the negative of a solution is again a solution.

For the sake of completeness we state some related results. Ličko and Švec [5] have shown that all solutions of (1) are oscillatory if and only if $\int^{\infty} s^{\gamma} q(s) d s=\infty$. Belohorec [2] has shown the following. If there exists a number $\beta, 0<\beta<(1-\gamma) / 2$, such that $f(t) t^{(3+\gamma) /(2+\beta)} \uparrow$ $K_{1}<\infty$, then all nontrivial solutions of (1) are nonoscillatory. If $f(t) t^{(3+\gamma) / 2} \downarrow K_{2}>0$, then (1) has both (nontrivial) oscillatory and nonoscillatory solutions. For similar results pertaining to the case $1<\gamma$, see $[1]$ and $[4]$.

We can now state our major result. Its proof will be preceded by three lemmas.

THEOREM. If $q(t) \in C^{\prime}[a, \infty), q(t)>0$ and $q^{\prime}(t) \leqq 0$ for $t \geqq a$ and if $\int^{\infty} s q(s) d s<\infty$, then (1) has no oscillatory solutions.

Lemma 1. Suppose that $\int^{\infty} s q(s) d s<\infty$ and let $K>0$ be given. Then

Presented to the Society November 9, 1968; received by the editors June 24,1968 . 
there is $a t_{0} \geqq a$ and a solution $y(t)$ of (1) defined on $\left[t_{0}, \infty\right)$ such that $K / 2 \leqq y(t) \leqq K$ for $t \geqq t_{0}$ and $\lim _{t \rightarrow \infty} y(t)=K$.

Proof. Our proof is a modification of a proof given in [1]. Consider the integral equation

$$
\psi(t)=K-\int_{t}^{\infty}(s-t) q(s)(\psi(s))^{\gamma} d s .
$$

Let $t_{0}$ be such that

$$
\int_{t_{0}}^{\infty}\left(s-t_{0}\right) q(s) d s<\min \left\{\left(K^{1-\gamma}\right) / 2,\left[\gamma(2 / K)^{1-\gamma}\right]^{-1}\right\} .
$$

To prove the lemma it suffices to show that (2) has a solution $\psi(t)$ such that $K / 2 \leqq \psi(t) \leqq K$.

Let $\psi_{0}(t) \equiv K, t \geqq t_{0}$, and

$$
\psi_{n+1}(t)=K-\int_{t}^{\infty}(s-t) q(s)\left(\psi_{n}(s)\right)^{\gamma} d s, \quad t \geqq t_{0} .
$$

Then $K / 2 \leqq \psi_{n}(t) \leqq K$ for $t \geqq t_{0}$. Note that $F(\psi)=\psi^{\gamma}$ satisfies a Lipschitz condition for $K / 2 \leqq \psi \leqq K$ with Lipschitz constant $\gamma(2 / K)^{1-\gamma}$. Therefore

$$
\left|\left(\psi_{n+1}(t)\right)^{\gamma}-\left(\psi_{n}(t)\right)^{\gamma}\right| \leqq \gamma(2 / K)^{1-\gamma}\left|\psi_{n+1}(t)-\psi_{n}(t)\right|
$$

for $t \geqq t_{0}$ and

$$
\left|\psi_{n+1}(t)-\psi_{n}(t)\right| \leqq \gamma(2 / K)^{1-\gamma} \max _{t \geq t_{0}}\left|\psi_{n}(t)-\psi_{n-1}(t)\right| \int_{t}^{\infty}(s-t) q(s) d s
$$

also for $t \geqq t_{0}$. This shows that $\psi_{n}(t) \rightarrow \psi(t)$ uniformly on $\left[t_{0}, \infty\right)$ and hence $\psi(t)$ is a solution of (2) satisfying $K / 2 \leqq \psi(t) \leqq K$ for $t \geqq t_{0}$.

Lemma 2. Suppose that $q(t) \in C^{\prime}[a, \infty), q(t)>0$ and $q^{\prime}(t) \leqq 0$ for $t \geqq a$. Let $y(t)$ be a nontrivial oscillatory solution of (1). Let $\left\{t_{n}\right\}$ be a sequence of consecutive relative maxima of $|y(t)|$ such that $n>m \Rightarrow t_{n}>t_{m}$. Then $\left|y\left(t_{n}\right)\right|$ is nondecreasing as $n$ increases and $\lim _{n \rightarrow \infty} t_{n}=\infty$.

Proof. Multiply (1) by $y^{\prime}(t) / q(t)$ and integrate from $t_{n}$ to $t_{n+1}$ to obtain

$$
\int_{t_{n}}^{t_{n+1}} \frac{\left(y^{\prime}(s)\right)^{2}}{2} \cdot \frac{q^{\prime}(s)}{(q(s))^{2}} d s+\frac{\left(y\left(t_{n+1}\right)\right)^{\gamma+1}}{\gamma+1}-\frac{\left(y\left(t_{n}\right)\right)^{\gamma+1}}{\gamma+1}=0 .
$$

Since $q^{\prime}(t) \leqq 0$, we get $\left|y\left(t_{n+1}\right)\right| \geqq\left|y\left(t_{n}\right)\right|$.

Note that $\lim _{n \rightarrow \infty} t_{n}=\infty$ is not immediate because global unique- 
ness for initial value problems does not hold in the case $\gamma<1$. Suppose that $\lim _{m \rightarrow \infty} t_{n}=t^{*}<\infty$. Since $|y(t)|$ is increasing at its relative maxima, we can apply the mean value theorem to get a sequence $\left\{s_{n}\right\} \rightarrow t^{*}$ such that $\lim _{n \rightarrow \infty}\left|y^{\prime}\left(s_{n}\right)\right|=\infty$. But this contradicts the fact that $y(t)$ exists on $[a, \infty)$.

LEMMA 3. Let $u(t), v(t), w(t)$ be solutions of (1) satisfying $0 \leqq u(t)$ $\leqq v(t) \leqq w(t)$ for $t^{\prime} \leqq t \leqq t^{\prime \prime}$. Define $\phi(t)$ by

$$
\phi(t)=(w-v)\left(v^{\prime}-u^{\prime}\right)-(v-u)\left(w^{\prime}-v^{\prime}\right) .
$$

Then $\phi\left(t^{\prime}\right) \geqq \phi\left(t^{\prime \prime}\right)$.

Proof. The statement and proof of this lemma are adapted from Lemma 1 of [6]. Note that in our case

$$
\left(v^{\gamma}-u^{\gamma}\right)(w-u) \geqq\left(w^{\gamma}-u^{\gamma}\right)(v-u) .
$$

Proof of Theorem. Suppose to the contrary that $y_{1}(t)$ is an oscillatory solution of (1). Let $\left\{t_{n}\right\}$ be the sequence of consecutive relative maxima of $\left|y_{1}(t)\right|$. Then $\lim _{n \rightarrow \infty} t_{n}=\infty$ and $0<\lim _{n \rightarrow \infty}\left|y_{1}\left(t_{n}\right)\right|$ $\equiv L \leqq \infty$ by Lemma 2 .

Let $0<K<L$ and let $y_{2}(t)$ be a solution of (1) such that $y_{2}(t) \uparrow K$ as $t \rightarrow \infty$ (by Lemma 1). Then we can find two points $t^{\prime}, t^{\prime \prime}$ such that the following situation occurs: $0<y_{1}\left(t^{\prime}\right)=y_{2}\left(t^{\prime}\right), 0<y_{1}\left(t^{\prime \prime}\right)=y_{2}\left(t^{\prime \prime}\right)$, and $0<y_{2}(t)<y_{1}(t)$ for $t^{\prime}<t<t^{\prime \prime}$. If we now set $u(t) \equiv 0, v(t)=y_{2}(t)$, and $w(t)=y_{1}(t)$, we see that $\phi\left(t^{\prime}\right)<\phi\left(t^{\prime \prime}\right) \quad(\phi(t)$ is defined in Lemma 3$)$. But this contradicts Lemma 3 . This proves the theorem.

REMARK. The question arises as to whether the conditions $q(t)>0$, $q^{\prime}(t) \leqq 0$ are necessary in the theorem. We conjecture that the weaker condition $q(t) \geqq 0$ is not sufficient. However, this weaker condition is sufficient for the following corollary.

CoRollary. If $\int^{\infty} s q(s) d s<\infty$ and if $y(t)$ is an oscillatory solution of (1), then $\lim _{t \rightarrow \infty} y(t)=\lim _{t \rightarrow \infty} y^{\prime}(t)=0$.

Proof. The fact that $\lim _{t \rightarrow \infty} y(t)=0$ follows from the proof of the theorem. Given $\epsilon>0$ pick $t_{0}$ so large that $\int_{t_{0}}^{\infty} q(s) d s<1$ and $|y(t)|^{\gamma}<\epsilon$ for $t \geqq t_{0}$. Since $y(t)$ is oscillatory we may suppose that $y^{\prime}\left(t_{0}\right)=0$. Therefore, by integrating (1) from $t_{0}$ to $t$ we get

$$
\left|y^{\prime}(t)\right| \leqq \epsilon \int_{t_{0}}^{t} q(s)<\epsilon, \quad t \geqq t_{0} .
$$

Since $\epsilon$ is arbitrary it follows that $\lim _{t \rightarrow \infty} y^{\prime}(t)=0$ (see also [2, Theorem 2]). 


\section{REFERENCES}

1. F. V. Atkinson, On second-order non-linear oscillations, Pacific J. Math. 5 (1955), 643-647.

2. Stefan Belohorec, On some properties of the equation $y^{\prime \prime}+f(x) y^{\alpha}(x)=0,0<\alpha<1$, Mat. Casopis Sloven. Akad. Vied. 17 (1967), 10-19.

3. Philip Hartman, Ordinary differential equations, Wiley, New York, 1964.

4. I. T. Kiguradze, On conditions for the oscillation of solutions of the equation $u^{\prime \prime}+a(t)|u|^{n} \operatorname{sgn} u=0$, Casopis Pest. Mat. 87 (1962), 492-495. (Russian)

5. Imrich Ličko and Marko Švec, Le charactère oscillatoire des solutions de l'équation $y^{(n)}+f(x) y^{\alpha}=0, n>1$, Czechoslovak Math. J. 88 (1963), 481-491.

6. R. A. Moore and Z. Nehari, Nonoscillation theorems for a class of nonlinear differential equations, Trans. Amer. Math. Soc. 93 (1959), 30-52.

University of Tennessee 